dowes of celomel, at tro hours interval, the stupor sradually subsiding, a black draught was given; moreral green motions quickly followed; and we suspended further trestment. Thirty hours from the onset of the convulsion, labour supervened, was quick and normal, and, to my child was very small, and lived but a few days. The patient continued to have pain in the head and drowsiness for a week or more. The mouth was decidedly sore the day after labour. The albumen gradually diminished; but, even after a long course of the tincture of sesquichloride of iron with quinine, it is doubtful if the urine ever became perfectly free from its presence. However, I at last lost sight of my patient.

Nine months have elapsed, and I am requested once more to call on Mrs. N. I find her now seven months advanced in pregnancy. She has her usual pallid, pasty look, together with an expression of careless apathy or stupidity in her countenance. She complains of pain in her head, with constant desire to sleep; and feels, as she expresses it, "holf tipsy". There is odema of the face, hands, and feet; the urine is very scanty, and loaded with albumen. Having seen the advanced stage of dropsy from Bright's disease arrested for a long period by mercurial action, I have thought it well to give her small doses of calomel ; these have just now slightly, but decidedly, affected her mouth. IIer state, however, at present continues precisely the same, and I am looking forward with no very hopeful prospect to the end.

Although albuminuria, in the latter months of gestation, is not uncommon, and a frequent cause of convulsions, yet $I$ apprehend that, in many of these cases, the abnormal state of the urine is solely dependent upon a temporary congestion of the renal organs from mechanical obstruction, precisely as the enormously swelled legs we occasionally see are due to pressure upon the iliac veins. I fear, however, that my patient's case cannot be admitted into this category; the child, as I have already said, in her former pregnancy, having been exceedingly small, and floating so freely in the liquor amnii (which was not superabundant) that no undue. pressure could possibly have been exerted. Moreover, she has previously borne twins, without any untoward symptoms.

The case is an interesting one, and possibly may elicit from some of the readers of the Jouras valuable suggestions.

\section{ON A NEW INSTRUMENT FOR VACCINATION: THE VACCINE INSITOR.}

By George Enward Nicholas, Esq., Wandsworth.

FaILURE of vaccination is very frequent. This is the candid acknowledgment of the whole profession. Without reference to the often and well explained causes of the failure of vaccination as a prophylactic when successfully performed, it must be admitted that it were more consistent with a state measure, and more creditable to medicine, that the operation should at least not carry with it any intrinsic means of affording umbrage to the vast amount of popular prejudice which exists against it. This antipathy on the part of the public, in fact, too frequently results from a want of immediate success in the operation itself, by which also much inconvenience occurs to the operator. This want of success has usually been ascribed to a want of dexterity and expertness; and it will be doubtless acknowledged that the performance of vaccination with the lancet, according to the usual plan, certainly requires a most dexterous hand to fulfil the generally admitted requirements; such as, that the cuticle alono be raised, and the cutis beneath exposed or arrived at, but not wounded, and that the puncture (?) be neither too wide, nor too deep, nor irregular, etc. To do this, observes a writer on the subject, requires "a hand as dexterous as ever fell to the lot of the beat of surgeons." We are not all the best of surgeons, but it is to be hoped we aro not all bunglers; and it is admitted that this kind of failure often occurs when the operation has been most carefully and scientifically performed. The blame lies mainly not with the operator but, as I have long since learned, with the instrument employed.

With the exception of a scarificator, and one or two other similarly barbarous instruments of torture, very rarely used, the lancet may be said to be the instrument in universal use, and has been so long so, that doubtless many will be very loth to discard it. Yet a little consideration will show, if every vaccinator has not already arrived at the fact, that the lancet is not at all well calculated for the service required of it; for, in consequence of its shape, it is ill adapted for the insertion of the lymph, and, however used, it cannot make other than an incised wound, which, when recent, is not the most favourable for absorption. A punctured wound alone is the one which is capable of insuring the greatest certainty of absorption.

To those who, like myself, have been ofteu inconvenienced by failures, but are unwilling to admit themselves guilty of inexpertuess, I beg to offer a means of repudiation of all blame in this respect, in the use of an instrument which I have contrived, by which are attained the greatest facility and rapidity of operating, together with what is most important, the greatest certainty of results. In regard to the latter, I noed only say that, during the last quarter, I have vaccinated with the instrument ninetysix cases continuously, with the occurrence of one failure only, that being of an adult previously vaccinated; that I am still using it; and, in short, that $I$ have experienced but two failures since I commenced to use it in March last.

This instrument, which I have rentured to call an "insitor" (of which a sketch, of full size, is subjoined), consists essentially of two bayonet-shaped processes, hinged at their base, where they are attached to a handle, and free at their points, which are extremely delicate, and so finely adjusted, as when pressed together to be capable of making a single puncture. The points are kept separated by a spring, the distance between them being regulated by a screw. When used, the instrument is lightly held between the finger and thumb, the handle lying in the palm of the hand; a little lymph is received between the points, the distance between which is adjusted in accordance with the viscosity of the lymph (usually from the one-sixtieth to the one-fiftieth of an inch); pressure is then applied by the finger and thumb to cause apposition of the points, and insertion is made. When this is done, the pressure is remitted; the points opening dilate the wound, and allow the lymph, which has flowed upwards during the continuance of the pressure, to return to the points. The instrument is now withdrawn; the tissue by its elasticity contracting round it effectually retains the lymph ; the instrument is wiped in the wound. Thus the insertion and retention of the lymph, and the most favourable wound for its absorption,


are insured.

The vaccine insitor can be obtained of Mr. Coxeter, 23, Grafton Street East, Tottenham Court Road, who has undertaken its manufacture. 